\title{
BIOPROSPECTING ENDOPHYTIC BACTERIA FOR BIOLOGICAL CONTROL OF COFFEE LEAF RUST
}

\author{
Humberto Franco Shiomi; Harllen Sandro Alves Silva; Itamar Soares de Melo; Flávia Vieira \\ Nunes; Wagner Bettiol* \\ Embrapa Meio Ambiente - Lab. de Microbiologia Ambiental, C.P. 69 - 13820-000 - Jaguariúna, SP - Brasil. \\ *Corresponding author <bettiol@cnpma.embrapa.br>
}

\begin{abstract}
Suppression of plant diseases due to the action of endophytic microorganisms has been demonstrated in several pathosystems. Experiments under controlled conditions involving endophytic bacteria isolated from leaves and branches of Coffea arabica $\mathrm{L}$ and Coffea robusta $\mathrm{L}$ were conducted with the objective of evaluating the inhibition of germination of Hemileia vastatrix Berk. \& Br., race II, urediniospores and the control of coffee leaf rust development in tests with leaf discs, detached leaves, and on potted seedling of cv. Mundo Novo. The endophytic bacterial isolates tested proved to be effective in inhibiting urediniospore germination and/or rust development, with values above $50 \%$, although the results obtained in urediniospore germination tests were inferior to the treatment with fungicide propiconazole. Endophytic isolates TG4-Ia, TF2-IIc, TF9-Ia, TG11-IIa, and TF7-IIa, demonstrated better coffee leaf rust control in leaf discs, detached leaves, and coffee plant tests. The endophytic isolates TG4-Ia and TF9-Ia were identified as Bacillus lentimorbus Dutky and Bacillus cereus Frank. \& Frank., respectively. Some endophytic bacterial isolates were effective in controlling the coffee leaf rust, although some increased the severity of the disease. Even though a relatively small number of endophytic bacteria were tested, promising results were obtained regarding the efficiency of coffee leaf rust biocontrol. These selected agents appears to be an alternative for future replacement of chemical fungicide.
\end{abstract}

Key words: Hemileia vastatrix, Coffea spp., Bacillus, biocontrol

\section{BIOPROSPECÇÃO DE BACTÉRIAS ENDOFÍTICAS COMO AGENTES DE BIOCONTROLE DA FERRUGEM DO CAFEEIRO}

RESUMO: Supressão de doenças de plantas por microrganismos endofíticos tem sido demonstrada em diversos patossistemas. Neste trabalho foram selecionados isolados de bactérias endofíticas de folhas e ramos de cafeeiro com potencial para o controle biológico da ferrugem do cafeeiro, pois é conhecido que esses microrganismos podem possuir essa característica. Bactérias endofíticas isoladas previamente de folhas e ramos de Coffea arabica L e Coffea robusta L foram avaliadas quanto ao seu potencial de biocontrole da ferrugem do café causada pelo fungo Hemileia vastatrix Berk. \& Br., raça 2 . As bactérias foram testadas para a inibição da germinação de urediniosporos do fungo e em bioensaios para o controle do desenvolvimento da ferrugem alaranjada do cafeeiro em discos de folhas, folhas destacadas e mudas da cv. Mundo Novo. Os isolados de bactérias endofíticas testados demonstraram eficácia na inibição da germinação de urediniosporos e/ou no desenvolvimento da ferrugem, com valores acima de $50 \%$, embora os resultados obtidos nos testes de germinação de urediniosporos tenham sido inferiores ao tratamento com propiconazole (testemunha padrão). Nos testes em discos de folhas, folhas destacadas e em plantas de cafeeiro, os isolados endofíticos TG4-Ia, TF2-IIc, TF9-Ia, TG11-IIa e TF7-IIa demonstraram melhor controle da ferrugem do cafeeiro. Os isolados endofíticos TG4-Ia e TF9-Ia foram identificados como Bacillus lentimorbus Dutky e Bacillus cereus Frank. \& Frank., respectivamente. De acordo com os resultados verifica-se que alguns isolados foram eficientes em controlar a ferrugem do cafeeiro, embora alguns tenham aumentado a severidade da doença. Apesar do número relativamente baixo de bactérias endofíticas testadas, resultados promissores foram obtidos em relação ao controle biológico da ferrugem, sendo que esses poderão no futuro apresentar uma alternativa aos fungicidas.

Palavras-chave: Hemileia vastatrix, Coffea spp., Bacillus, controle biológico

\section{INTRODUCTION}

Beneficial endophytic microorganisms comprise especially fungi and bacteria that colonize internal plant tissues without causing visible damage to their hosts (Petrini, 1991). They are different from phytopathogenic microorganisms because they are not detrimental, do not cause diseases to plants, and are distinct from epiphytic microorganisms which live on the surface of plant organs and tissues (Hallmann et al., 
1997). Endophytic bacteria are able to penetrate and become systemically disseminated in the host plant, actively colonizing the apoplast (Quadt-Hallmann et al., 1997b), conducting vessels (Hallmann et al., 1997), and occasionally the intracellular spaces (QuadtHallmann et al., 1997a). This colonization presents an ecological niche, similar to that occupied by plant pathogens, and this endophytic bacteria can, therefore, act as biological control agents against pathogens (Hallmann et al., 1997).

In this sense, the suppression of plant diseases due to the action of endophytic microorganisms has been demonstrated in several pathosystems (Narisawa et al., 1998; Lima et al., 1994). Several mechanisms may control this suppression, either directly on the pathogen inside the plant by antibiosis (Sturz et al., 1998) and competition for nutrients (Mari et al., 1996), or indirectly by induction of plant resistance response (M’Piga et al., 1997).

The coffee leaf rust caused by Hemileia vastatrix is the main disease in coffee, causing yield losses of 35 to $40 \%$, on average. Control is basically achieved by fungicides. In 2000, in Brazil, the use of fungicides in coffee stood for 3,680 $t$ of active ingredient (Campanhola \& Bettiol, 2003). Therefore, alternatives to control coffee leaf rust must be sought. The objective of this work was to select endophytic bacteria isolates from coffee leaves and branches, with biocontrol potential against coffee leaf rust, by means of inhibition assays of urediniospores germination and control of coffee leaf rust in tests with leaf discs, detached leaves, and on potted seedling of cv Mundo Novo.

\section{MATERIAL AND METHODS}

\section{Endophytic bacteria}

Isolates from leaves and branches (Nunes, 2004), of Coffea arabica and Coffea robusta plants from Pedreira, Mococa, and Pindorama counties, State of São Paulo, Brazil (Table 1), were maintained in the culture colletion of the Laboratório de Microbiologia Ambiental, Embrapa Meio Ambiente, in sterile distilled water (Castellani, 1967). Forty bacterial isolates were evaluated regarding their capacity to inhibit the germination of $H$. vastatrix urediniospores, and 44 isolates were used to control coffee leaf rust in leaf discs, detached leaves, and seedlings of C. arabica, cv. Mundo Novo.

\section{Urediniospore germination}

The endophytic bacteria isolates were cultivated on nutrient agar medium (Peptone $5 \mathrm{~g}$; meat extract $3 \mathrm{~g}$; agar $15 \mathrm{~g}$; distilled water $1000 \mathrm{~mL}$ ) for 24 hours at $28 \pm 2^{\circ} \mathrm{C}$, and then transferred with to slants containing sterilized, distilled water. Samples were then shaken vigorously to obtain a homogeneous cell suspension, which was standardized to an optical density of $\mathrm{A}_{550}=0.1$. Urediniospores of $H$. vastatrix, race II, were collected from coffee leaves containing lesions obtained from plants in "Centro de Café e Plantas Tropicais, Instituto Agronômico de Campinas", and stored in a container with sodium dichromate (relative humidity $52 \% ; 7 \pm 2^{\circ} \mathrm{C}$ ). Urediniospores were suspended in water at a concentration of $1.0 \mathrm{mg} \mathrm{mL}^{-1}$ using a magnetic stirrer for 5 minutes. A $15.0 \mu \mathrm{L}$ aliquot of this suspension, and a $15.0 \mu \mathrm{L}$ aliquot of the endophytic bacteria suspension were then transferred to microscope slide, mixed and enclosed within plastic boxes containing a layer of foam saturated with water, and sealed with glass plates to maintain high relative humidity. After incubation for six hours $\left(22 \pm 1^{\circ} \mathrm{C}\right)$ in the dark, the germination was interrupted by adding 15.0 $\mu \mathrm{L}$ of lactophenol cotton blue dye onto each droplet, and examined under light microscope. The percentage of germinated urediniospores $(10$ fields at $200 \times$ magnification) was calculated. Urediniospores with germ tubes of at least one half of the length of their larger diameter were considered germinated. Trials were set up in a completely randomized experimental design $(n=4)$. The experiment was repeated and the means were used for statistical analysis. Sterilized water and propiconazole (Tilt ${ }^{\circledR} \mathrm{CE} ; 1.2 \mu \mathrm{L}$ of the commercial product per $\mathrm{mL}$ of water) were used as controls.

\section{Leaf discs}

Discs of young and completely developed leaves of $C$. arabica cv. Mundo Novo plants were removed with a $2.0 \mathrm{~cm}$ diameter cork punch and placed into plastic boxes, abaxial surface facing up, over a layer of foam saturated with water (Eskes, 1989). Using a micro-pipet, $25.0 \mu \mathrm{L}$ of the endophytic bacteria suspension were applied on the leaf discs, 72 and 24 hours before, after, and simultaneously with the same volume of $H$. vastatrix urediniospores suspension (1.0 $\left.\mathrm{mg} \mathrm{mL} \mathrm{m}^{-1}\right)$. After inoculation, boxes were covered with glass plates and incubated in the dark for 24 hours. Then the boxes were maintained under $12 \mathrm{~h}$ photoperiod, 500-1000 lux, $22 \pm 2^{\circ} \mathrm{C}$, and approximately $100 \%$ relative humidity. The experiment was set on completely randomized design $(n=3)$, represented by nine leaf discs each. Severity of the disease was evaluated 30 days after inoculation, using a rating scale from 1 to 5 , according to the percentage of leaf area with lesions $(1=0 \% ; 2=1-25 \% ; 3=26-50 \% ; 4=51-75 \%$; and $5 \geq 75 \%$ of leaf area with lesions). The WallerDuncan $(\alpha=0.05)$ test was used to compare the lowest and the highest mean values of lesions percentage for each treatment. 
Table 1 - Effect of endophytic bacterial isolates from coffee plants on the germination of Hemileia vastatrix urediniospores.

\begin{tabular}{|c|c|c|}
\hline Isolate & Source(Species-plant part-loction) & $\%$ of germinationof $\mathrm{H}$. vastratrix \\
\hline Water & - & $54.4 \mathrm{a}^{1}$ \\
\hline T F11-III a & Coffea arabica - leaf - Pindorama, SP & $48.6 \mathrm{ab}(10.7)$ \\
\hline T F7-I a & Coffea robusta - leaf - Pindorama, SP & $48.2 \mathrm{ab}(11.4)$ \\
\hline T F9-I b & C. robusta - leaf - Pindorama, SP & $48.1 \mathrm{ab}(11.5)$ \\
\hline T G6-I b & C. robusta - stem - Mococa, SP & 47.7 abc (12.3) \\
\hline A F2-I b & C. robusta - leaf - Pedreira, SP & $47.3 \mathrm{abc}(13.1)$ \\
\hline T G10-II d & C.arabica - leaf - Pindorama, SP & 46.2 abcd (15.1) \\
\hline T F2-II a & C. robusta - leaf - Mococa, SP & 41.2 bcde $(24.2)$ \\
\hline T G5-II b & C. robusta - stem - Mococa, SP & 40.8 bcde $(25)$ \\
\hline T F9-I a & C. robusta - leaf - Pindorama, SP & 39.4 bcdef $(27.5)$ \\
\hline T F3-II a & C. arabica - leaf - Pedreira, SP & 39.3 bcdefg $(27.8)$ \\
\hline T G5-III c & C. robusta - stem - Mococa, SP & 39.2 bcdefg $(27.9)$ \\
\hline T F12-I a & C.arabica - leaf - Pindorama, SP & 37.3 cdefgh $(31.5)$ \\
\hline T F4-II a & C. robusta - leaf - Mococa, SP & 36.3 defgh (33.2) \\
\hline T G7-III e & C. robusta - stem - Pindorama, SP & 33.6 efghi $(38.2)$ \\
\hline T G7-I c & C. robusta - stem - Pindorama, SP & 33.3 efghi $(38.8)$ \\
\hline A F7-III a & C. robusta - leaf - Pindorama, SP & 33.2 efghi (39) \\
\hline T G8-III b & C. robusta - stem - Pindorama, SP & 33.1 efghi (39.1) \\
\hline T F7-II b & C. robusta - leaf - Pindorama, SP & 32.6 efghij (40) \\
\hline T F5-III a & C. robusta - leaf - Mococa, SP & 31.7 efghij (41.6) \\
\hline T F10-III a & C.arabica - leaf - Pindorama, SP & 30.2 efgijk (44.5) \\
\hline T F8-I a & C. robusta - leaf - Pindorama, SP & 30.2 fghijkl (44.5) \\
\hline T F9-I c & C. robusta - leaf - Pindorama, SP & 29.9 fghijkl (44.9) \\
\hline T F7-I b & C. robusta - leaf - Pindorama, SP & 29.6 fghijkl (45.5) \\
\hline T G10-III c & C.arabica - leaf - Pindorama, SP & 29.5 fghijkl (45.8) \\
\hline T F10-II a & C.arabica - leaf - Pindorama, SP & 29.4 fghijkl (46) \\
\hline T F7-III a & C. robusta - leaf - Pindorama, SP & 29.1 fghijkl (46.5) \\
\hline T F7-II a & C. robusta - leaf - Pindorama, SP & 28.8 ghijkl (47.1) \\
\hline T G4-II a & C. robusta - stem - Mococa, SP & 28.6 hijkl (47.4) \\
\hline T G12-III a & C. arabica - stem - Pindorama, SP & 28.3 hijkl (48) \\
\hline T G4-I a & C. robusta - stem - Mococa, SP & 28.1 hijkl (48.2) \\
\hline T F7-III b & C. robusta - leaf - Pindorama, SP & 27.9 hijkl (48.6) \\
\hline T G12-II c & C. arabica - stem - Pindorama, SP & 27.4 hijkl (49.6) \\
\hline T F2-I c & C. arabica - leaf - Pedreira, SP & $24.9 \mathrm{ijkl}(54.1)$ \\
\hline T G8-II a & C. robusta - stem - Pindorama, SP & 23.9 ijklm (54.3) \\
\hline T F9-I d & C. robusta - leaf - Pindorama, SP & $23.8 \mathrm{ijk} \operatorname{lm}(56)$ \\
\hline T F4-III a & C. robusta - leaf - Mococa, SP & $23.6 \mathrm{ijk} \operatorname{lm}(56.6)$ \\
\hline T G11-II a & C. arabica - stem - Pindorama, SP & $22.1 \mathrm{jk} \operatorname{lm}(59.3)$ \\
\hline T F9-I a F & C. robusta - leaf - Pindorama, SP & $21.3 \mathrm{k} \operatorname{lm}(60.8)$ \\
\hline A F7-II b & C. robusta - leaf - Pindorama, SP & $20.7 \operatorname{lm}(61.9)$ \\
\hline T F2-II c & C. arabica - leaf - Pedreira, SP & $14.3 \mathrm{~m} \mathrm{(73.7)}$ \\
\hline Propiconazole & - & $2.5 \mathrm{n}(95.4)$ \\
\hline
\end{tabular}

\section{Detached leaves}

Ten isolates of endophytic bacteria used in this experiment were selected according to results observed in the two previous tests. The treatments consisted of a bacterial suspension $\left(\mathrm{A}_{550}=0.1\right)$ sprayed on completely developed coffee leaves $(C$. arabica $\mathrm{cv}$. Mundo Novo), 72 and 24 hours before, after and simulta- neously with the inoculation of the urediniospore suspension. The coffee leaves were placed in plastic boxes, abaxial surface facing up, over a layer of foam saturated with water, covered with a glass plate and incubated as described. The experimental design was randomized blocks $(n=3)$, each replicate consisting of three leaves. Inoculation was performed using a 
sprayer attached to a compressor, pressure $10 \mathrm{lb} \mathrm{in}^{-2}$. Following inoculation, the boxes were covered and placed in the dark for 24 hours, at $22 \pm 2^{\circ} \mathrm{C}$. Treatments were evaluated 21 days after inoculation, by counting the number of lesions per leaf. Means were compared by Tukey test $(\alpha=0.05)$.

\section{Coffee plants}

The same endophytic bacterial isolates used in the detached leaves were used in this study. Coffee seedlings (C. arabica cv. Mundo Novo) susceptible to all $H$. vastatrix strains were obtained from "Centro de Café e Plantas Tropicais, Instituto Agronômico de Campinas", and transplanted into plastic pots containg $5 \mathrm{~L}$ of Red Yellow Latosol, sifted through $1.0 \mathrm{~cm}^{2}$ mesh sieve and mixed with $2.0 \mathrm{~kg}$ of lime, $5.0 \mathrm{~kg}$ of simple superphosphate, and $0.5 \mathrm{~kg}$ of potassium chloride per $\mathrm{m}^{3}$ of soil. The bacterial suspensions $\left(\mathrm{A}_{550}=\right.$ 0.1 ) were manually sprayed to the foliage until runoff. The urediniospore suspension was applied with a sprayer attached to a compressor, pressure $10 \mathrm{lb} \mathrm{in}^{-2}$. After inoculation with the $H$. vastatrix urediniospore suspension $\left(1.0 \mathrm{mg} \mathrm{mL}^{-1}\right)$, plants were incubated in the dark for 48 hours at $22 \pm 2^{\circ} \mathrm{C}, 100 \%$ relative humidity, and then transferred to a greenhouse. Plants were irrigated daily and after 30 days the number of lesions per inoculated leaf was evaluated. Sterilized water was used as control. Trial was set up in a randomized blocks design $(\mathrm{n}=3)$, with two plants per pot and, means compared by Tukey test $(\alpha=0.05)$.

The most effective isolates were identified based on cell membrane fatty acid contents, analyzed in a gas chromatograph, using microbial identification software (MIDI, Sherlock ${ }^{\circledR}$ TSBA Library version 5.0, Microbial ID, Newark, DE, USA). Isolates with a similarity index of 0.6 or higher were considered positively identified.

\section{RESULTS AND DISCUSSION}

Twenty three out of the 40 endophytic bacteria isolates tested for their capacity of inhibiting $H$. vastatrix urediniospore germination inhibited germination in more than $40 \%$ (Table 1), irrespective of source (coffee species or plant organ), in relation to the control (water). In addition, deformations of the germination tube that were detrimental compared to normal development, were observed. All bacterial isolates were statistically inferior to the propiconazole.

In the leaf disc assay, the endophytic bacterium TG4-Ia reduced disease severity in all application intervals tested. Control levels were above $63 \%$ when applied at 72 and 24 hours before or simultaneously to fungal pathogen inoculation (Table 2). Other isolates were also effective in reducing rust development in the leaf disc tests, but with less intensity. In general, lower control levels were observed when the bacterium was applied after pathogen inoculation. Some isolates increased severity of the disease, especially TF4-IIa, which increased the severity of lesions by $64 \%$ when applied 72 hours before the pathogen. Only TG11-IIa was effective in inhibiting in vitro urediniospore germination (Table 1), and in reducing the percentage of leaf area with lesions in coffee leaf discs. Isolates TF2IIc, TF3-IIa, TF7-Ib, TF7-IIa, TF9-IIa, AF7-IIIa, TG4Ia, TG4-IIa, TG10-IIIc, and TG11-Iia, were selected for further studies on detached leaves and on coffee plants.

In the detached leaves assay, the most prominent isolates were TF7-Ib, TF9-Ia, TF3-IIa, TG10-IIIc, and TF7-IIa, which showed significant control in all application intervals tested (Table 3). Even though the endophytic bacterium TF9-Ia has yielded the best control $(62.0 \%)$, its performance was not reproducible on coffee plants. There was a decline in the number of isolates able of reducing severity of the disease, as the interval between the presence of the biocontrol agents and the pathogen decreased.

In the test with coffee plants, the endophytic bacteria were not effective in controlling coffee leaf rust when applied after inoculation of the pathogen (Table 4). Isolates TF2-IIc, TF7-IIa, TG4-Ia, and TG11-IIa were effective when applied either 72 and 24 before or concurrently with the pathogen (Table 4).

Endophytes TG4-Ia, TF9-Ia, TF2-IIc, and TF7IIa, identified as Bacillus lentimorbus, Bacillus cereus, Clavibacter michiganensis subsp. michiganensis Smith, and Klebsiella pneumoniae Schroeter, respectively, showed the best performance. The other endophytes were identified as Bacillus sp. (TF3-IIa), Klebsiella pneumoniae (TF7-Ib), Pandorae pnomenusa Coenye at al. (AF7-IIIa), Kocuria kristinae Kloos et al. (TG4-IIa), Cedecea davisae Grimont et al. (TG10IIIc), and Acinetobacter calcoaceticus Beijerinck (TG11-IIa).

The efficiency of certain endophytic bacteria isolates in controlling coffee leaf rust can vary according to the moment of biocontrol agent application. In general, the endophytes were more effective when applied 72 and 24 hours before and concurrently with the inoculation of $H$. vastatrix urediniospores. Similar results were obtained by Bettiol et al. (1994) and Bettiol \& Várzea (1992); these authors registered reductions in the percentage of leaves with lesions and in the number of lesions per leaf rating $60 \%$ and $100 \%$, by spraying different concentrations of non-endophytic Bacillus subtilis-based products 72 and 24 hours before the application of $H$. vastatrix urediniospores, on coffee 
Table 2 - Percentage of leaf area with lesions caused by coffee leaf rust, in cv. "Mundo Novo" leaf discs, submitted to suspensions of endophytic bacteria isolates 72 hours before and after, 24 hours before and after, and concurrently with the inoculation of Hemileia vastatrix.

\begin{tabular}{|c|c|c|c|c|c|}
\hline Isolates & - 72 hours & - 24 hours & 0 hours & +24 hours & +72 hours \\
\hline Water & 10.8 bcdefgh & $13.6 \mathrm{ab}$ & 10.7 efghij & 12.2 abcde & $14.3 \mathrm{ab}$ \\
\hline TF 1-IIa & 7.8 ghi (27.9) & 8.4 abcdefg (37.9) & 5.2 klmno $(50.7)$ & 12.4 abcde & 11.4 abcd (19.8) \\
\hline TF2-Ic & 9.8 defgh $(9.0)$ & 9.9 abcdef (26.8) & $16.2 \mathrm{abc}$ & 12.1 abcde (1.4) & 11.0 abcd (11.1) \\
\hline TF2-IIc & 11.2 bcdefgh & $3.0 \mathrm{fg}(77.6)$ & 2.4 no $(77.0)$ & 11.6 abcdef (11.7) & $12.6 \mathrm{abcd}(12.1)$ \\
\hline TF3-IIa & $15.3 \mathrm{abc}$ & 6.9 bcdefg (49.4) & 1.2 o $(88.1)$ & 9.3 bcdef ( 9.3$)$ & $14.6 \mathrm{a}$ \\
\hline TF4-IIa & $17.8 \mathrm{a}$ & 8.8 abcdefg (35.2) & 11.0 defghij & $14.0 \mathrm{abcd}$ & $14.1 \mathrm{ab}(1.2)$ \\
\hline TF4-IIIa & 12.8 bcdef & 9.4 abcdef (31.0) & 11.0 defghij & 11.2 abcdef (8.4) & - \\
\hline TF5-IIIa & 13.8 abcde & 10.1 abcde (25.4) & 12.8 abcdefgh & 12.7 abcde & $12.3 \mathrm{abcd}(14.2)$ \\
\hline TF7-Ia & 14.0 abcde & 10.7 abcd (21.0) & 13.6 abcdefgh & 11.4 abcdef (6.9) & 13.8 abcd (3.2) \\
\hline TF7-Ib & 9.9 defgh (8.5) & $2.1 \mathrm{~g}(84.1)$ & 6.4 jklmn (39.6) & $12.2 \mathrm{abcde}$ & $13.2 \mathrm{abcd}(7.3)$ \\
\hline TF7-IIa & $12.0 \mathrm{bcdefgh}$ & 7.1 bcdefg (47.9) & 4.3 lmno (59.3) & $13.6 \mathrm{abcd}$ & $14.0 \mathrm{ab}(1.9)$ \\
\hline TF7-IIb & 9.8 defgh (8.9) & 9.9 abcdef (26.8) & 14.1 abcdefgh & $13.6 \mathrm{abcd}$ & $14.0 \mathrm{abc}(2.1)$ \\
\hline TF7-IIIa & 13.4 abcde & 11.6 abcd (14.5) & $17.1 \mathrm{ab}$ & 11.8 abcdef (3.3) & $13.8 \mathrm{abcd}(3.2)$ \\
\hline TF7-IIIb & $14.6 \mathrm{abcd}$ & 7.1 bcdefg (47.3) & 12.6 abcdefgh & 7.3 def (40) & $12.7 \mathrm{abcd}(10.7)$ \\
\hline TF8-Ia & 13.2 abcde & $10.4 \mathrm{abcd}(23.2)$ & $17.7 \mathrm{a}$ & 10.2 abcdef (16.7) & $13.2 \mathrm{abcd}(7.8)$ \\
\hline TF8-IIa & $14.0 \mathrm{abcd}$ & 11.1 abcd (18.4) & 14.1 abcdefgh & 11.8 abcdef (3.7) & $9.3 \mathrm{abcd}(35.1)$ \\
\hline TF9-Ia & 7.4 hi (31.7) & 6.0 cdefg (55.9) & 2.3 no $(78.1)$ & 12.3 abcde & $10.0 \mathrm{abcd}(30.0)$ \\
\hline TF9-Ia F & 9.1 efgh (16.1) & 11.6 abcd (14.4) & $16.1 \mathrm{abcd}$ & 11.6 abcdef (4.8) & $10.4 \mathrm{abcd}(27.3)$ \\
\hline TF9-Ib & 8.1 fghi (24.9) & 11.4 abcd (15.7) & 15.8 abcde & 13.0 abcde & 12.9 abcd (9.8) \\
\hline TF9-Ic & $11.7 \mathrm{bcdefgh}$ & 8.9 abcdefg (34.4) & 10.0 fghijk (6.2) & $14.5 \mathrm{ab}$ & 12.9 abcd (9.5) \\
\hline TF9-Id & $10.5 \mathrm{cdefgh}$ & 7.0 bcdefg (48.4) & 10.4 fghijk (2.4) & 10.5 abcdef (13.6) & $9.3 \mathrm{abcd}(35.1)$ \\
\hline TF $10-$ Ia & $12.2 \mathrm{bcdefgh}$ & 9.7 abcdef (28.3) & 12.0 bcdefghi & 12.6 abcde & 11.7 abcd (17.9) \\
\hline TF 10-IIa & 12.4 bcdefg & 12.4 abcd (8.9) & 10.3 fghijk (3.3) & $16.7 \mathrm{a}$ & $10.7 \mathrm{abcd}(24.8)$ \\
\hline TF10-IIIa & $11.7 \mathrm{bcdefgh}$ & $12.6 \mathrm{abcd}(7.1)$ & 11.3 cdefghij & $14.5 \mathrm{ab}$ & $10.8 \mathrm{abcd}(24.3)$ \\
\hline TF11-IIIa & 10.9 bcdefgh & $14.2 \mathrm{a}$ & 15.1 abcdef & $14.3 \mathrm{abc}$ & $13.3 \mathrm{abcd}(6.7)$ \\
\hline TF12-Ia & $15.3 \mathrm{abc}$ & $12.0 \mathrm{abcd}(11.3)$ & 11.8 cdefghi & 10.0 abcdef (18.0) & $9.0 \mathrm{abcd}(36.6)$ \\
\hline AF2-Ib & $12.3 \mathrm{bcdefg}$ & 12.9 abc (5) & 10.2 fghijk (4.3) & 9.4 bcdef (22.5) & $8.3 \mathrm{bcd}(41.8)$ \\
\hline AF 7-IIb & 10.5 cdefgh $(2.7)$ & 10.0 abcdef (26.4) & 11.9 cdefghi & 11.5 abcdef (6.0) & $7.8 \mathrm{~cd}(7.8)$ \\
\hline AF7-IIIa & 11.3 bcdefgh & 8.6 abcdefg $(36.6)$ & $7.7 \mathrm{ijk} \operatorname{lm}(28.1)$ & 9.9 abcdef (18.5) & $7.6 \mathrm{~d}(7.7)$ \\
\hline TG4-Ia & 3.9 i (63.8) & 3.1 efg (76.7) & 1.6 no $(85)$ & 7.5 cdef (38.5) & $8.3 \mathrm{bcd}(8.3)$ \\
\hline TG4-IIa & 10.5 cdefgh (3.3) & $13.7 \mathrm{ab}$ & 11.5 cdefghij & 8.8 bcdef (28.1) & 11.3 abcd (11.4) \\
\hline TG5-IIb & 9.95 defgh $(8.4)$ & 5.9 cdefg $(56.5)$ & 9.2 hijkl (14.1) & 10.7abcdef (12.1) & $10.2 \mathrm{abcd}(10.2)$ \\
\hline TG5-IIIc & 11.5 bcdefgh & 7.6 abcdefg (43.9) & 9.8 ghijk (8.3) & 9.4 bcdef (23) & 11.9 abcd (16.9) \\
\hline TG6-Ib & $11.3 \mathrm{bcdefgh}$ & 9.1 abcdefg (32.9) & 12.1 bcdefghi & 11.0 abcdef (9.9) & $12.0 \mathrm{abcd}(16.2)$ \\
\hline TG7-Ic & 13.7 abcde & 11.9 abcd (12.7) & 11.6 cdefghij & 12.8 abcde & 11.0 abcd (23.0) \\
\hline TG7-IIIe & $14.1 \mathrm{abcd}$ & 9.8 abcdef (27.8) & 14.7 abcdefg & 11.7 abcdef (4.2) & $10.6 \mathrm{abcd}(25.5)$ \\
\hline TG8-IIa & $15.5 \mathrm{ab}$ & 9.7 abcdef (28.2) & 12.2 bcdefghi & 10.9 abcdef $(10.5)$ & 9.6 abcd (9.6) \\
\hline TG8-IIIb & $15.1 \mathrm{abc}$ & 8.4 abcdefg (38.1) & 11.8 cdefghi & 9.7 bcdef (20.3) & 11.6 abcd (11.7) \\
\hline TG8-IIIc & 13.4 abcde & $12.3 \mathrm{abcd}(9.2)$ & 10.9 efghij & 9.9 abcdef (18.8) & 11.5 abcd (11.5) \\
\hline TG10-IId & $15.4 \mathrm{abc}$ & 10.0 abcdef (26.2) & 10.5 fghij (2.1) & 6.3 ef (47.8) & $12.0 \mathrm{abcd}(12.0)$ \\
\hline TG10-IIIc & $14.5 \mathrm{abcd}$ & 5.7 defg (57.6) & 3.7 mno $(65.0)$ & 9.2 bcdef $(24.6)$ & 10.6 abcd (10.7) \\
\hline TG11-IIa & $12.1 \mathrm{bcdefgh}$ & $3.0 \mathrm{fg}(77.9)$ & 2.2 no $(79.3)$ & $4.9 \mathrm{f}(59.3)$ & 11.4 abcd (11.5) \\
\hline TG11-IIb & 13.4 abcde & 10.9 abcd (20.3) & 11.9 bcdefghi & 8.4 bcdef $(31.0)$ & $10.0 \mathrm{abcd}(10.0)$ \\
\hline TG12-IIc & 11.3 bcdefgh & 8.8 abcdefg (34.9) & 11.2 cdefghij & 9.8 bcdef (19.6) & $10.1 \mathrm{abcd}(10.2)$ \\
\hline TG12-IIIa & 12.4 bcdefg & 9.9 abcdef (27.0) & 10.2 fghijk (4.6) & 8.6 bcdef $(8.6)$ & 11.4 abcd (20.4) \\
\hline
\end{tabular}

${ }^{1}$ Means followed by the same letter do not differ among themselves (Waller-Duncan 5\%). Values refer to means of three replicates, with nine discs each. The numbers between parentheses indicate the percentage of control of the disease relative to the control treatment. TF1-IIa and TF10-Ia were isolated from leaves and TG11-IIb from stem of Coffea arabica from Pindorama. TG8-IIIc and TF8-IIa were isolated from stem and leaves, from C. robusta from Pindorama, respectively. 
Table 3 - Effect of endophytic bacteria applied 72 and 24 hours before and after, and concurrently with the inoculation of Hemileia vastatrix urediniospores, on the number of rust lesions per leaf in detached leaves of coffee (Coffea arabica) cv. Mundo Novo.

\begin{tabular}{|c|c|c|c|c|c|}
\hline Isolate/Species & - 72 hours & -24 hours & 0 hours & +24 hours & +72 hours \\
\hline Water - control & 216.8 a 1 & $195.4 \mathrm{a}$ & 200.2 a & $234.1 \mathrm{a}$ & $232.4 \mathrm{a}$ \\
\hline $\begin{array}{l}\text { TF2-IIc - Clavibacter } \\
\text { michiganensis subsp. michiganensis }\end{array}$ & 84.8 b $(60)$ & $86.2 \mathrm{~b}(56)$ & $109.7 \mathrm{ab}(45)$ & 162.0 abcd (31) & 110.9 c (52) \\
\hline TF3-IIa - Bacillus sp. & $90.1 \mathrm{~b}(58)$ & 87.1 b $(55)$ & $8.2 \mathrm{~b}(56)$ & 113.1 bcd (51) & 101.0 c $(56)$ \\
\hline TF7-Ib - Klebsiella pneumoniae & $84.6 \mathrm{~b}(61)$ & $72.7 \mathrm{~b}(63)$ & $88.3 \mathrm{~b}(56)$ & $107.7 \mathrm{~cd}(54)$ & $137.6 \mathrm{bc}(41)$ \\
\hline TF7-IIa - Klebsiella pneumoniae & 89.4 b (58) & $81.0 \mathrm{~b}(58)$ & $74.0 \mathrm{~b}(63)$ & $101.3 \mathrm{~cd}(56)$ & 97.6 c $(58)$ \\
\hline TF9-Ia - Bacillus cereus & $82.9 \mathrm{~b}(62)$ & 80.9 b (58) & $90.4 \mathrm{~b}(55)$ & 128.9 bcd (45) & 119.4 bc (48) \\
\hline AF7-IIIa - Pandorae pnomenusa & 110.3 b (49) & $85.1 \mathrm{~b}(56)$ & $124.6 \mathrm{ab}(38)$ & $98.1 \mathrm{~d}(58)$ & $134.8 \mathrm{bc}(42)$ \\
\hline TG4-Ia - Bacillus lentimorbus & 99.9 b (53) & $112.4 \mathrm{~b}(42)$ & $113.2 \mathrm{ab}(43)$ & $111.8 \mathrm{bcd}(52)$ & 228.0 a (2) \\
\hline TG4- IIa - Kocuria cristinae & $130.2 \mathrm{~b}(40)$ & $94.8 \mathrm{~b}(51)$ & $152.3 \mathrm{ab}(24)$ & $207.6 \mathrm{ab}(11)$ & $197.1 \mathrm{ab}(15)$ \\
\hline TG10-IIIc - Cedecea davisae & $83.7 \mathrm{~b}(61)$ & 80.8 b (59) & $102.1 \mathrm{~b}(49)$ & 165.1 abcd (29) & 140.6 bc (39) \\
\hline $\begin{array}{l}\text { TG11-IIa - Acinetobacter } \\
\text { calcoaceticus }\end{array}$ & $160.3 \mathrm{ab}(26)$ & $145.2 \mathrm{ab}(26)$ & $114.1 \mathrm{ab}(43)$ & $196.9 \mathrm{abc}(16)$ & $234.0 \mathrm{a}$ \\
\hline
\end{tabular}

${ }^{1}$ Means followed by the same letter do not differ among themselves (Tukey 5\%). In detached leaf tests, values refer to means of three replicates, with three leaves each; in seedling tests, values refer to the means of 10 replicates. The numbers between parentheses indicate the percentage of control of the disease relative to the control treatment.

Table 4 - Effect of endophytic bacteria applied 72 and 24 hours before and after, and concurrently with the inoculation of Hemileia vastatrix urediniospores, on the number of rust lesions per leaf of coffee plants (Coffea arabica) cv. Mundo Novo.

\begin{tabular}{|c|c|c|c|c|c|}
\hline Isolate/Species & - 72 hours & -24 hours & 0 hours & +24 hours & +72 hours \\
\hline Water - control & $143.2 \mathrm{abc}$ & $155.3 \mathrm{a}$ & $148.3 \mathrm{a}$ & $130.6 \mathrm{a}$ & $134.4 \mathrm{a}$ \\
\hline $\begin{array}{l}\text { TF2-IIc - Clavibacter } \\
\text { michiganensis subsp. michiganensis }\end{array}$ & 76.8 ef (43) & 95.8 c (38) & $31.1 \mathrm{~cd}(79)$ & $146.8 \mathrm{a}$ & $141.4 \mathrm{a}$ \\
\hline TF3-IIa - Bacillus sp. & 134.7 abcd (6) & $110.2 \mathrm{abc}(29)$ & $79.1 \mathrm{~b}(46)$ & $162.1 \mathrm{a}$ & $136.1 \mathrm{a}$ \\
\hline TF7-Ib - Klebsiella pneumoniae & $174.4 \mathrm{a}$ & $153.4 \mathrm{ab}(1)$ & 71.9 bc $(51)$ & $149.7 \mathrm{a}$ & 133.9 a \\
\hline TF7-IIa - Klebsiella pneumoniae & 88.0 cdef (38) & 77.8 c $(50)$ & 88.9 b (40) & 124.0 a (5) & $139.7 \mathrm{a}$ \\
\hline TF9-Ia - Bacillus cereus & 110.0 bcdef $(23)$ & 99.4 bc (36) & $107.5 \mathrm{ab}(27)$ & 129.9 a & 132.7 a $(1)$ \\
\hline AF7-IIIa - Pandorae pnomenusa & 122.9 abcde (14) & 107.8 abc $(31)$ & 74.9 bc (49) & $133.3 \mathrm{a}$ & $153.5 \mathrm{a}$ \\
\hline TG4-Ia - Bacillus lentimorbus & $61.7 \mathrm{f}(57)$ & 73.8 c (52) & $22.2 \mathrm{~d}(85)$ & 117.6 a (9) & 120.2 a $(10)$ \\
\hline TG4- IIa - Kocuria cristinae & 120.5 abcde (16) & 113.6 abc $(27)$ & $115.6 \mathrm{ab}(22)$ & $151.8 \mathrm{a}$ & 144.6 a \\
\hline TG10-IIIc - Cedecea davisae & $154.1 \mathrm{ab}$ & 80.3 c (48) & 91.8 b (38) & $134.1 \mathrm{a}$ & $144.5 \mathrm{a}$ \\
\hline $\begin{array}{l}\text { TG11-IIa - Acinetobacter } \\
\text { calcoaceticus }\end{array}$ & $83.2 \mathrm{def}(42)$ & 79.6 c (49) & 89.4 b (39) & 127.2 a (2) & $135.2 \mathrm{a}$ \\
\hline
\end{tabular}

${ }^{1}$ Means followed by the same letter do not differ among themselves (Tukey 5\%). In detached leaf tests, values refer to means of three replicates, with three leaves each; in seedling tests, values refer to the means of 10 replicates. The numbers between parentheses indicate the percentage of control of the disease relative to the control treatment.

plants, cv. Catuaí. The fact that the endophytic isolates showed activity when applied before the pathogen suggests that these isolates may act by antibiosis, lysis of pathogen structures, competition, or induction of systemic resistance in the host.

Kim et al. (2002) reported that the bacterium $B$. lentimorbus produces the antifungal substances alpha- and beta-glucosidase, with an inhibitory action against the development of Botrytis cinerea Pers.:Fr, while Sadfi et al. (2001) reported that this bacterium is capable of releasing volatile substances that contribute to the inhibition of Fusarium sambucinum Fuckel in potato tubers. Several authors demonstrated that $B$. cereus can promote growth in various plant species such as tomato (Simon et al., 2001), and wheat (Ryder et al., 1999). In addition, it can actively penetrate the 
tissues and disseminate inter- and intracellularly within the host, protecting it from $F$. sambucinum, in potatoes, by producing fungitoxic substances (Chérif et al., 2003). Also, B. cereus can produce various chitinases, active against several plant pathogens, such as $F$. sambucinum (Sadfi et al., 2001), Rhizoctonia solani Kühn (Ryder et al., 1999), Helminthosporium solani Dur. \& Mont. (Martinez et al., 2002), Sclerotium rolfsii Sacc., Fusarium oxysporum Schl., and Pythium aphanidermatum (Edson) Fitzp. B. cereus has also been reported as endophytic in cotton (Gossypium hirsutum L), sweet corn (Zea mays L), and citrus plants (Citrus spp.) by Di Fiore \& Del Gallo (1995).

It could thus be speculated that there were more than one mode of action of those endophytic bacteria in the control of coffee leaf rust. Inhibitory action against $H$. vastatrix in urediniospore germination was shown in specific essays, tests with leaf discs, with detached leaves, and with coffee plants at different application intervals.

The endophytic association of bacteria of the genera Clavibacter and Klebsiella with some agronomic crops, such as corn, grapevine, rice, cotton, and some crucifers was reported by Lodewyckx et al. (2002). However, reports on the application of species of the above-mentioned genera dealing with control of pathogens are scarce in the literature. C. michiganensis subsp. michiganensis is a phytopathogenic species (Agrios, 1997), while K. pneumoniae can be found in hospitals causing infections in humans (MartinsLoureiro et al., 2001). These characteristics can create barriers to the application of these bacteria in bioassays seeking plant disease biocontrol agents. No reports were found in biological control on the application of the other tested endophytes.

Properties of some endophytic isolates in increasing coffee leaf rust severity shall be highlighted. According to Musson (1994), some endophytic organisms can behave as non-pathogenic in a given host, and as pathogenic in another. Cameron (1970) found that Pseudomonas spp. isolates obtained from healthy cherry tissues proved to be phytopathogenic in further tests. This author suggested that the endophyte condition could be one of the forms of survival and escape against surface phytosanitary treatments. Additionally, Whitesides \& Spotts (1991) found Pseudomonas syringae van Hall isolates from pear tree roots, which were not pathogenic neither to cherry nor to pear trees, and suggested that the internal tissues of pear trees could function as inoculum reservoir to other plants. Thus, the ability of colonizing internal plant tissues could be visualized as a survival mechanism of plant pathogenic bacteria, since they exist in a protected position (Leben, 1981). This fact has biological impor- tance, because demonstrates that the interactions with endophytes could have economic importance related to both the control and to the expansion of plant diseases.

Even though a relatively small number of endophytic bacteria were tested, promising results were obtained regarding the selection of coffee leaf rust biocontrol agents. Further field studies must be conducted to analyze the real potential of endophytic bacteria in field conditions. Studies are also needed to determine the modes of action of those bacteria, the population density of the applied endophytes, and the best form of introduction into the host. The evaluation of the effects of agrochemicals on endophytic bacteria have also to be considered, since those can not only stimulate isolates that are beneficial to rust control but can also select those that increase severity of the disease.

\section{REFERENCES}

AGRIOS, G.N. Plant pathology. 4.ed. New York: Academic Press, 1997. $635 \mathrm{p}$.

BETTIOL, W.; VARZEA, V.M.P. Controle biológico da ferrugem (Hemileia vastatrix) do cafeeiro com Bacillus subtilis em condições controladas. Fitopatologia Brasileira, v.17, p.91-95, 1992.

BETTIOL, W.; SAITO, M.L.; BRANDÃO, M.S.B. Controle da ferrugem do cafeeiro com produtos à base de Bacillus subtilis. Summa Phytopathologica, v.20, p.119-122, 1994.

CAMERON, H.R. Pseudomonas content of cherry trees. Phytopathology, v.60, p.1343-1346, 1970.

CAMPANHOLA, C.; BETTIOL, W. Panorama sobre o uso de agrotóxicos no Brasil. In: CAMPANHOLA, C.; BETTIOL, W. (Ed.). Métodos alternativos de controle fitossanitário. Jaguariúna: Embrapa Meio Ambiente, 2003. p.13-51.

CASTELLANI, A. Maintenance and cultivation of common pathogenic fungi in sterile distilled water, for the researches. Journal of Tropical Medicine and Hygiene, v.70, p.181-184, 1967.

CHÉRIF, M.; SADFI, N.; OUELLETTE, G.B. Ultrastructure of in vivo interactions of the antagonistic bacteria Bacillus cereus X16 and B. thuringiensis 55T with Fusarium roseum var. sambucinum, the causal agent of potato dry rot. Phytopathologia Mediterranea, v.42, p.4154, 2003.

DI FIORE, S.; DEL GALLO, M. Endophytic bacteria: their possible role in the host plants. In: FENDRIK, I.; DEL GALLO, M.; VANDERLEYDEN, J.; DE ZAMAROCZY, M. (Ed.) Azospirilum VI and related microrganisms. Berlin: Springer-Verlag, 1995. p.169-187.

ESKES, A.B. Resistance. In: KUSHALAPPA, A.C.; ESKES, A.B. (Ed.). Coffee rust: epidemiology, resistance, and management. Boca Raton: CRC Press, 1989. p.171-291.

FAETH, S.H.; HAMMON, K.E. Fungal endophytes in oak trees: experimental analyses of interaction with leafminers. Ecology, v.78, p.820-827, 1997.

HALLMANN, J.; QUADT-HALLMANN, A.; MAHAFFEE, W.F.; KLOEPPER, J.W. Bacterial endophytes in agricultural crops. Canadian Journal of Microbiology, v.43, p.895-914, 1997.

KIM, K.J.A.; YANG, Y.J.; KIM, J. Production of alpha-glucosidase inhibitor by beta-glucosidase inhibitor producing Bacillus lentimorbus B-6. Journal of Microbiology and Biotechnology, v.12, p.895-900, 2002.

LEBEN, C. How plant-pathogenic bacteria survive. Plant Disease, v.65, p.633-637, 1981. 
LIMA, G.; IPPOLITO, A.; NIGRO, F.; SALERNO, M. Atempts in the biological Control of citrus mal secco (Phoma tracheiphila) using endophytic bacteria. Difesa Delle Piante, v.17, p.43-49, 1994.

LODEWYCKX, C.; VANGROSVELD, J.; PORTEOUS, F.; MOORE, E.R.B.; TAGHAVI, S.; MEZGEAY, M.; VAN DER LELIE, D. Endophytic bacteria and their potential applications. Critical Reviews in Plant Science, v.21, p.583-606, 2002.

MARI, M.; GUIZZARDI, M.; PRATELLA, G.C. Biological control of gray mold in pears by antagonistic bacteria. Biological Control, v.7, p.30-37, 1996.

MARTINEZ, C.; MICHAUD, M.; BELANGER, R.R.; TWEDDELL, R.J. Identification of soils suppressive against Helminthosporium solani, the causal agent of potato silver scurf. Soil Biology and Biochemistry, v.34, p.1861-1868, 2002.

MARTINS-LOUREIRO, M.; MORAES, B.A.; MENDONÇA, V.L.F.; ROCHA-QUADRA, M.R.; SANTOS-PINHEIRO, G.; DUTRAASENSI, M. Molecular epidemiology of extended-spectrum bLactamase-producing Klebsiella pneumoniae isolated from neonatal intensive care unit patients involved in hospital infections cases in Rio de Janeiro, Brazil. Revista Latinoamericana de Microbiologia, v.43, p.88-95, 2001.

M'PIGA, P.; BÉLANGER, R.R.; PAULITZ, T.C.; BENHAMOU, N. Increased resistance to Fusarium oxysporum f. sp. radicis-lycopersici in tomato plants treated with the endophytic bacterium Pseudomonas fluorescens strain 63-28. Physiological and Molecular Plant Pathology, v.50, p.301-320, 1997.

MUSSON, G. Ecology and effects of endophytic bacteria in plants. Auburn: Auburn University, 1994. 114p. (Ms Thesis).

NARISAWA, K.; TOKUMASU, S.; HASHIBA, T. Suppression of clubroot formation in chinese cabbage by the root endophytic fungus, Heteroconium chaetospira. Plant Pathology, v.47, p.206-210, 1998.

NUNES, F.V. Isolamento e identificação de bactérias endofíticas do café (Coffea arábica e Coffea robusta) e seu potencial biotecnológico. São Paulo: USP/Interunidades, 2004. (Dissertação - Mestrado).
PETRINI, O. Fungal endophyte of tree leaves. In: ANDREWS, J.; HIRANO, S.S. (Ed.). Microbial ecology of leaves. New York: Spring Verlag, 1991. p.179-197.

QUADT-HALLMANN, A.; HALLMANN, J.; KLOEPPER, J.W. Bacterial endophytes in cotton: localization and interaction with other plant-associated bacteria. Canadian Journal of Microbiology, v.43, p.254-259, 1997a.

QUADT-HALLMANN, A.; BENHAMOU, N.; KLOEPPER, J.W. Bacterial endophytes in cotton: mechanisms of entering the plant. Canadian Journal of Microbiology, v.43, p.577-582, 1997b.

RYDER, M.H.; YAN, Z.; TERRACE, T.E.; ROVIRA, A.D.; TANG, W.H.; CORRELL, R.L. Use of strains of Bacillus isolated in China to suppress take-all and Rhizoctonia root rot, and promote seedling growth of glasshouse-grown wheat in Australian soils. Soil Biology and Biochemistry, v.31, p.19-29, 1999.

SADFI, N.; CHERIF, M.; FLISS, I.; BOUDABBOUS, A.; ANTOUN, $\mathrm{H}$. Evaluation of bacterial isolates from salty soils and Bacillus thuringiensis strains for the biocontrol of Fusarium dry rot of potato tubers. Journal of Plant Pathology, v.83, p.101-118, 2001.

SIMON, H.M.; SMITH, K.P.; DODSWORTH, J.A.; GUENTHNER, B.; HANDELSMAN, J.; GOODMAN, R.M. Influence of tomato genotype on growth of inoculated and indigenous bacteria in the spermosphere. Applied and Environmental Microbiology, v.67, p.514-520, 2001.

STURZ, A.V.; CHRISTIE, B.R.; MATHESON, B.G. Association of bacterial endophyte populations from red clover and potato crops with potential for beneficial allelopathy. Canadian Journal of Microbiology, v.44, p.162-167, 1998.

WHITESIDES, S.K.; SPOTTS, R.A. Frequency, distribution and characteristics of endophytic Pseudomonas syringae in pear trees. Phytopathology, v.81, p.453-457, 1991.

Received April 01, 2004

Accepted December 28, 2005 